\title{
Description of the diploid chromosome set of Triatoma pintodiasi (Hemiptera, Triatominae)
}

\author{
K.C.C. Alevi ${ }^{1}$, F.F.F. Moreira ${ }^{2}$, J. Jurberg ${ }^{2}$ and M.T.V. Azeredo-Oliveira ${ }^{1}$ \\ ${ }^{1}$ Laboratório de Biologia Celular, Departamento de Biologia, \\ Instituto de Biociências, Letras e Ciências Exatas, \\ Universidade Estadual Paulista "Júlio de Mesquita Filho", São José do Rio Preto, \\ SP, Brasil \\ ${ }^{2}$ Laboratório Nacional e Internacional de Referência em Taxonomia de Triatomíneos, \\ Instituto Oswaldo Cruz, Rio de Janeiro, RJ, Brasil \\ Corresponding author: K.C.C. Alevi \\ E-mail: kaiochaboli@hotmail.com \\ Genet. Mol. Res. 15 (2): gmr.15026343 \\ Received June 15, 2015 \\ Accepted December 22, 2015 \\ Published April 25, 2016 \\ DOI http://dx.doi.org/10.4238/gmr.15026343
}

\begin{abstract}
Triatoma pintodiasi has been described and recently grouped in the Rubrovaria subcomplex. T. pintodiasi was initially compared to $T$. carcavalloi by staining and subsequently identified as $T$. circummaculata. However, after thorough examination, it was observed to be a cryptic species of $T$. circummaculata, and was described based on morphological features, morphometric data, and biochemical patterns of hemolymph. Thus, this paper aims to describe the karyotype of, and spermatogenesis in, T. pintodiasi, in order to elucidate the reproductive biology and taxonomy of the species. Sex chromosomes of T. pintodiasi formed a heteropyknotic chromocenter, and compaction of chromatin was observed during prophase. However, in contrast to observations in T. carcavalloi and T. circummaculata, in T. pintodiasi it was observed individualization of the sex chromosomes. The diploid chromosome set of the species $2 n=22(20 A+X Y)$ is described through analysis of metaphases I and II. Initial cytogenetic characteristics of $T$. pintodiasi are described and the observed differences in the chromocenter
\end{abstract}


are suggested as a possible cytotaxonomic tool. To gain a better understanding of the specific status of this cryptic species, however, we emphasize the need for further cytogenetic, molecular, biological, and biogeographical analysis, in addition to experimental hybrid crosses with other species of the Rubrovaria subcomplex.

Key words: Karyotype; Spermatogenesis; Rubrovaria subcomplex; Triatoma pintodiasi

\section{INTRODUCTION}

The triatomines are insects of great epidemiological interest. The 148 species currently described (Abad-Franch et al., 2013; Alevi et al., 2013a; Jurberg et al., 2013; Poinar Jr., 2013) are potential vectors of the protozoan Trypanosoma cruzi, the etiologic agent of Chagas disease. Triatomines are also of biological interest because their cells have several peculiarities in comparison to cells of other eukaryotes, such as holocentric chromosomes (Panzera et al., 1996), inverted meiosis for sex chromosomes (Gómez-Palacio et al., 2008), and persistence of nucleolar material during meiosis (Tartarotti and Azeredo-Oliveira, 1999; Alevi et al., 2014a). In addition, triatomines are of evolutionary interest because their origins (monophyletic or polyphyletic) have not been conclusively determined (Tartarotti et al., 2006; Hwang and Weirauch, 2012).

For over 50 years, these hematophagous insects have been grouped into both complexes and specific subcomplexes (de Lucena, 1970; Dujardin et al., 2002; Schofield and Galvão, 2009; Justi et al., 2014). Although species complexes and subcomplexes are not formally recognized as taxonomic ranks, they should be monophyletic (Justi et al., 2014).

Schofield and Galvão (2009) proposed that the Rubrovaria subcomplex is composed of the species Triatoma carcavalloi, T. circummaculata, T. klugi, T. limai, T. oliveirai, and T. rubrovaria. The species of the Rubrovaria subcomplex were initially grouped through morphological analyses: T. rubrovaria and $T$. carcavalloi categorized in the Rubrovaria subcomplex; T. circummaculata and T. limai in the Circummaculata complex; and T. klugi in the Oliverai complex (Dujardin et al., 2002). Almeida et al. (2009) grouped T. circummaculata in subcomplex Rubrovaria based on phylogenetic and molecular data, and Gardim et al. (2013) and Justi et al. (2014) identified the subcomplex Rubrovaria as monophyletic. However, new approaches, such as experimental hybrid crosses and cytogenetic analyzes, are important and necessary to assist in understanding the evolutionary development of these vectors.

A new species, T. pintodiasi, has recently been described and grouped in Rubrovaria subcomplex (Jurberg et al., 2013). T. pintodiasi was initially compared to T. carcavalloi based on staining, but was found to be morphologically smaller. Subsequently, $T$. pintodiasi was classified as T. circummaculata. However, after thorough examination, it was observed to be a cryptic species of $T$. circummaculata, and was described based on morphological features, morphometric data, and biochemical patterns of hemolymph (Jurberg et al., 2013). The process of spermatogenesis and the karyotype of $T$. pintodiasi are described in the present study, with the aim of acquiring a detailed understanding of the reproductive biology and taxonomy of the species. In addition, a chromosomal review of all triatomine species is presented with karyotype descriptions. 


\section{MATERIAL AND METHODS}

At least three adult males each of T. pintodiasi, T. carcavalloi, and T. circummaculata were analyzed. The specimens of $T$. pintodiasi were provided by the National Laboratory and International Reference on Taxonomy of Triatominae, Instituto Oswaldo Cruz, Rio de Janeiro, Brazil, and the species of T. carcavalloi and T. circummaculata were provided by Insectarium of Triatominae, FCFAR/UNESP, Araraquara, São Paulo, Brazil. Seminiferous tubules of $T$. pintodiasi, T. carcavalloi, and T. circummaculata were isolated, shredded, smashed, and set on a slide in liquid nitrogen. They were then stained by the cytogenetic technique using lactoacetic orcein as outlined by De Vaio et al. (1985), with modifications according to Alevi et al. (2012a). The biological material was analyzed by a Jenaval light microscope (Zeiss) coupled to a digital camera and an image analyzer Axio Vision LE 4.8 (Copyright ${ }^{\circ}$ 2006-2009 Carl $^{-2}$ Zeiss Imaging Solutions $\mathrm{GmbH}$ ). The images were subjected to $1000 \mathrm{X}$ magnification. All karyotypes described in the literature are presented in Table 1.

\section{RESULTS}

The early stages of meiosis in spermatogenesis were described by means of classical cytogenetic analysis. Compaction of chromatin (Figure 1A-C and 2A) was observed during prophase, with the formation of chiasma between autosomes (Figure 1C).

The sex chromosomes of $T$. pintodiasi formed a heteropyknotic chromocenter (Figure 1A-C and 2A); however, individualization remained throughout prophase. This observation was not consistent with that for T. carcavalloi (Figure 2B) and $T$. circummaculata (Figure 2C). The diploid chromosome set of the species $2 \mathrm{n}=22(20 \mathrm{~A}+$ $\mathrm{XY}$ ) (Figure 1C and D, respectively), was determined by analysis of metaphases I and II. In both metaphases I and II, the Y sex chromosomes of T. pintodiasi were larger and more heteropyknotic (Figure 1D and E; arrows).

Analysis of the species presented in Table 1, revealed that two species have 21 chromosomes $\left(2 \mathrm{n}=18+\mathrm{X}_{1} \mathrm{X}_{2} \mathrm{Y}\right) ; 51$ species have 22 chromosomes $(2 \mathrm{n}=20+\mathrm{XY}) ; 30$ species have 23 chromosomes $\left(2 \mathrm{n}=20+\mathrm{X}_{1} \mathrm{X}_{2} \mathrm{Y}\right)$; four species have 24 chromosomes $(2 \mathrm{n}=$ $\left.20+\mathrm{X}_{1} \mathrm{X}_{2} \mathrm{X}_{3} \mathrm{Y}\right)$; and only one species has 25 chromosomes $\left(2 \mathrm{n}=22+\mathrm{X}_{1} \mathrm{X}_{2} \mathrm{Y}\right)$.

\section{DISCUSSION}

In cryptic speciation, the resulting species show great morphological similarity. Specific tools are necessary to differentiate between cryptic species. It was possible to distinguish the sex chromosomes in a heteropyknotic chromocenter during meiotic prophase in $T$. pintodiasi. This feature is quite peculiar in the subfamily Triatominae, since most triatomines have only one heteropyknotic chromocenter, as observed in T. carcavalloi and $T$. circummaculata (Panzera et al., 1998).

T. pintodiasi presented a karyotype consisting of 22 chromosomes, consistent with that observed for all species of the Rubrovaria subcomplex (Ueshima, 1966; Panzera et al., 1996; Alevi et al., 2013a). Although this number can vary from 21 to 25 chromosomes, 22 has been described as the modal number of chromosomes for the Triatominae subfamily (Ueshima, 1966) (Table 1). Ueshima (1966) also proposed that Triatominae has 22 chromosomes $(20 \mathrm{~A}+\mathrm{XY})$. 
Table 1. Review of all triatomine species with karyotype descriptions.

\begin{tabular}{|c|c|c|c|}
\hline No. & Triatomines & Karyotype & Described by: \\
\hline & Tribe Alberproseniini & & \\
\hline & Genus Alberprosenia & & \\
\hline 1 & Alberprosenia goyovargasi & Not described & \\
\hline \multirow[t]{3}{*}{2} & Alberprosenia malheiroi & Not described & \\
\hline & Tribe Bolboderini & & \\
\hline & Genus Belminus & & \\
\hline 3 & Belminus corredori & Not described & \\
\hline 4 & Belminus costaricensis & Not described & \\
\hline 5 & Belminus ferroae & Not described & \\
\hline 6 & Belminus herreri & Not described & \\
\hline 7 & Belminus laportei & Not described & \\
\hline 8 & Belminus peruvianus & Not described & \\
\hline 9 & Belminus pittieri & Not described & \\
\hline \multirow[t]{2}{*}{10} & Belminus rugulosus & Not described & \\
\hline & Genus Bolbodera & & \\
\hline \multirow[t]{2}{*}{11} & Bolbodera scabrosa & Not described & \\
\hline & Genus Microtriatoma & & \\
\hline 12 & Microtriatoma borbai & Not described & \\
\hline \multirow[t]{2}{*}{13} & Microtriatoma trinidadensis & Not described & \\
\hline & Genus Parabelminus & & \\
\hline 14 & Parabelminus carioca & Not described & \\
\hline \multirow[t]{3}{*}{15} & Parabelminus yurupucu & Not described & \\
\hline & Tribe Cavernicolini & & \\
\hline & Genus Cavernicola & & \\
\hline 16 & Cavernicola lenti & Not described & \\
\hline \multirow[t]{3}{*}{17} & Cavernicola pilosa & Not described & \\
\hline & Tribe Linshcosteini & & \\
\hline & Genus Linshcosteus & & \\
\hline 18 & Linshcosteus carnifex & Not described & \\
\hline 19 & Linshcosteus chota & Not described & \\
\hline 20 & Linshcosteus confumus & Not described & \\
\hline 21 & Linshcosteus costalis & Not described & \\
\hline 22 & Linshcosteus kali & Not described & \\
\hline \multirow[t]{3}{*}{23} & Linshcosteus karupus & Not described & \\
\hline & Tribe Rhodniini & & \\
\hline & Genus Psammolestes & & \\
\hline 24 & Psammolestes arthuri & Not described & \\
\hline 25 & Psammolestes coreodes & $2 n=22(20 A+X Y)$ & Schreiber and Pellegrino, 1950 \\
\hline \multirow[t]{2}{*}{26} & Psammolestes tertius & $2 n=22(20 A+X Y)$ & Panzera et al., 1998 \\
\hline & Genus Rhodnius & & \\
\hline 27 & Rhodnius amazonicus & Not described & \\
\hline 28 & Rhodnius barretti & Not described & \\
\hline 29 & Rhodnius brethesi & $2 n=22(20 A+X Y)$ & Panzera et al., 1998 \\
\hline 30 & Rhodnius colombiensis & $2 n=22(20 A+X Y)$ & Dujardin et al., 2002 \\
\hline 31 & Rhodnius dalessandroi & Not described & \\
\hline 32 & Rhodnius domesticus & $2 n=22(20 A+X Y)$ & Dujardin et al., 2002 \\
\hline 33 & Rhodnius ecuadoriensis & $2 n=22(20 A+X Y)$ & Panzera et al., 1998 \\
\hline 34 & Rhodnius milesi & $2 n=22(20 A+X Y)$ & Panzera et al., 2010 \\
\hline 35 & Rhodnius montenegrensis & $2 n=22(20 A+X Y)$ & Alevi et al., 2015a \\
\hline 36 & Rhodnius nasutus & $2 n=22(20 A+X Y)$ & Pérez et al., 1992 \\
\hline 37 & Rhodnius neglectus & $2 n=22(20 A+X Y)$ & Barth, 1956 \\
\hline 38 & Rhodnius neivai & $2 n=22(20 A+X Y)$ & Koshy, 1979a \\
\hline 39 & Rhodnius pallescens & $2 n=22(20 A+X Y)$ & Panzera et al., 1996 \\
\hline 40 & Rhodnius paraensis & Not described & \\
\hline 41 & Rhodnius pictipes & $2 n=22(20 A+X Y)$ & Koshy, 1979b \\
\hline 42 & Rhodnius prolixus & $2 n=22(20 A+X Y)$ & Schreiber and Pellegrino, 1950 \\
\hline 43 & Rhodnius robustus & $2 n=22(20 A+X Y)$ & Koshy, $1979 b$ \\
\hline 44 & Rhodnius stali & $2 n=22(20 A+X Y)$ & Dujardin et al., 2002 \\
\hline 45 & Rhodnius zeledoni & Not described & \\
\hline
\end{tabular}

Continued on next page 
Table 1. Continued.

\begin{tabular}{|c|c|c|c|}
\hline No. & Triatomines & Karyotype & Described by: \\
\hline & Tribe Triatomini & & \\
\hline & Genus Dipetalogaster & & \\
\hline \multirow[t]{2}{*}{46} & Dipetalogaster maxima & $2 n=22(20 A+X Y)$ & Ueshima, 1966 \\
\hline & Genus Eratyrus & & \\
\hline 47 & Eratyrus cuspidatus & $2 n=23\left(20 A+X_{1} X_{2} Y\right)$ & Dujardin et al., 2002 \\
\hline \multirow[t]{2}{*}{48} & Eratyrus mucronatus & $2 \mathrm{n}=23\left(20 \mathrm{~A}+\mathrm{X}_{1} \mathrm{X}_{2} \mathrm{Y}\right)$ & Dujardin et al., 2002 \\
\hline & Genus Hermanlentia & & \\
\hline \multirow[t]{2}{*}{49} & Hermanlentia matsunoi & Not described & \\
\hline & Genus Meccus & & \\
\hline 50 & Meccus bassolsae & $2 n=23\left(20 A+X_{1} X_{2} Y\right)$ & Dujardin et al., 2002 \\
\hline 51 & Meccus longipennis & $2 \mathrm{n}=23\left(20 \mathrm{~A}+\mathrm{X}_{1} \mathrm{X}_{2} \mathrm{Y}\right)$ & Panzera et al., 1996 \\
\hline 52 & Meccus mazzottii & $2 \mathrm{n}=23\left(20 \mathrm{~A}+\mathrm{X}_{1} \mathrm{X}_{2} \mathrm{Y}\right)$ & Panzera et al., 1996 \\
\hline 53 & Meccus pallidipennis & $2 n=23\left(20 A+X_{1} X_{2} Y\right)$ & Ueshima, 1966 \\
\hline 54 & Meccus phyllosomus & $2 n=23\left(20 A+X_{1} X_{2} Y\right)$ & Dujardin et al., 2002 \\
\hline \multirow[t]{2}{*}{55} & Meccus picturatus & $2 n=23\left(20 A+X_{1} X_{2} Y\right)$ & Panzera et al., 1996 \\
\hline & Genus Mepraia & & \\
\hline 56 & Mepraia gajardoi & $2 \mathrm{n}=23\left(20 \mathrm{~A}+\mathrm{X}_{1} \mathrm{X}_{2} \mathrm{Y}\right)$ & Frías et al., 1998 \\
\hline 57 & Mepraia parapatrica & $2 \mathrm{n}=23\left(20 \mathrm{~A}+\mathrm{X}_{1} \mathrm{X}_{2} \mathrm{Y}\right)$ & Frías-Lasserre, 2010 \\
\hline \multirow[t]{2}{*}{58} & Mepraia spinolai & $2 n=23\left(20 A+X_{1} X_{2} Y\right)$ & Panzera et al., 1998 \\
\hline & Genus Nesotriatoma & & \\
\hline 59 & Nesotriatoma bruneri & $2 n=23\left(20 A+X_{1} X_{2} Y\right)$ & Panzera et al., 2010 \\
\hline 60 & Nesotriatoma flavida & $2 \mathrm{n}=23\left(20 \mathrm{~A}+\mathrm{X}_{1} \mathrm{X}_{2} \mathrm{Y}\right)$ & Dujardin et al., 2002 \\
\hline \multirow[t]{2}{*}{61} & Nesotriatoma obscura & Not described & \\
\hline & Genus Paratriatoma & & \\
\hline \multirow[t]{2}{*}{62} & Paratriatoma hirsuta & $2 n=22(20 A+X Y)$ & Ueshima, 1966 \\
\hline & Genus Panstrongylus & & \\
\hline 63 & Panstrongylus chinai & $2 n=23\left(20 A+X_{1} X_{2} Y\right)$ & Crossa et al., 2002 \\
\hline 64 & Panstrongylus diasi & Not described & \\
\hline 65 & Panstrongylus geniculatus & $2 n=23\left(20 A+X_{1} X_{2} Y\right)$ & Crossa et al., 2002 \\
\hline 66 & Panstrongylus guentheri & Not described & \\
\hline 67 & Panstrongylus hispaniolae & Not described & \\
\hline 68 & Panstrongylus howardi & $2 \mathrm{n}=23\left(20 \mathrm{~A}+\mathrm{X}_{1} \mathrm{X}_{2} \mathrm{Y}\right)$ & Panzera et al., 2010 \\
\hline 69 & Panstrongylus humeralis & Not described & \\
\hline 70 & Panstrongylus lenti & Not described & \\
\hline 71 & Panstrongylus lignarius & $2 \mathrm{n}=23\left(20 \mathrm{~A}+\mathrm{X}_{1} \mathrm{X}_{2} \mathrm{Y}\right)$ & Crossa et al., 2002 \\
\hline 72 & Panstrongylus lutzi & $2 \mathrm{n}=24\left(20 \mathrm{~A}+\mathrm{X}_{1} \mathrm{X}_{2} \mathrm{X}_{3} \mathrm{Y}\right)$ & Santos, 2010 \\
\hline 73 & Panstrongylus megistus & $2 \mathrm{n}=21\left(18 \mathrm{~A}+\mathrm{X}_{1} \mathrm{X}_{2} \mathrm{Y}\right)$ & Schreiber and Pellegrino, 1950 \\
\hline 74 & Panstrongylus mitarakaensis & Not described & \\
\hline 75 & Panstrongylus rufotuberculatus & $2 n=23\left(20 A+X_{1} X_{2} Y\right)$ & Crossa et al., 2002 \\
\hline \multirow[t]{2}{*}{76} & Panstrongylus tupynambai & $2 n=23\left(20 A+X_{1} X_{2} Y\right)$ & Panzera et al., 1998 \\
\hline & Genus Triatoma & & \\
\hline 77 & Triatoma amicitiae & Not described & \\
\hline 78 & Triatoma arthurneivai & $2 n=22(20 A+X Y)$ & Dujardin et al., 2002 \\
\hline 79 & Triatoma baratai & $2 n=22(20 A+X Y)$ & Alevi et al., 2015b \\
\hline 80 & Triatoma barberi & $2 n=23\left(20 A+X_{1} X_{2} Y\right)$ & Ueshima, 1966 \\
\hline 81 & Triatoma bolivari & Not described & \\
\hline 82 & Triatoma boliviana & Not described & \\
\hline 83 & Triatoma bouvieri & Not described & \\
\hline 84 & Triatoma brailovskyi & Not described & \\
\hline 85 & Triatoma brasiliensis & $2 n=22(20 A+X Y)$ & Schreiber and Pellegrino, 1950 \\
\hline 86 & Triatoma b. macromelanosoma & $2 n=22(20 A+X Y)$ & Panzera et al., 2000 \\
\hline 87 & Triatoma breyeri & Not described & \\
\hline 88 & Triatoma carcavalloi & $2 n=22(20 A+X Y)$ & Dujardin et al., 2002 \\
\hline 89 & Triatoma carrioni & Not described & \\
\hline 90 & Triatoma cavernicola & Not described & \\
\hline 91 & Triatoma circummaculata & $2 n=22(20 A+X Y)$ & Panzera et al., 1998 \\
\hline 92 & Triatoma costalimai & $2 \mathrm{n}=22(20 \mathrm{~A}+\mathrm{XY})$ & Dujardin et al., 2002 \\
\hline 93 & Triatoma deaneorum & Not described & \\
\hline 94 & Triatoma delpontei & $2 n=22(20 A+X Y)$ & Ueshima, 1966 \\
\hline
\end{tabular}




\section{Table 1. Continued.}

\begin{tabular}{|c|c|c|c|}
\hline No. & Triatomines & Karyotype & Described by: \\
\hline 95 & Triatoma dimidiata & $2 \mathrm{n}=23\left(20 \mathrm{~A}+\mathrm{X}_{1} \mathrm{X}_{2} \mathrm{Y}\right)$ & Panzera et al., 1996 \\
\hline 96 & Triatoma dispar & Not described & \\
\hline 97 & Triatoma eratyrusiformis & $2 n=24\left(20 A+X_{1} X_{2} X_{3} Y\right)$ & Ueshima, 1966 \\
\hline 98 & Triatoma garciabesi & $2 n=22(20 A+X Y)$ & Panzera et al., 1997 \\
\hline 99 & Triatoma gerstaeckeri & $2 n=23\left(20 A+X_{1} X_{2} Y\right)$ & Ueshima, 1966 \\
\hline 100 & Triatoma gomeznunezi & Not described & \\
\hline 101 & Triatoma guasayana & $2 n=22(20 A+X Y)$ & Panzera et al., 1996 \\
\hline 102 & Triatoma guazu & $2 \mathrm{n}=22(20 \mathrm{~A}+\mathrm{XY})$ & Dujardin et al., 2002 \\
\hline 103 & Triatoma hegneri & $2 n=23\left(20 A+X_{1} X_{2} Y\right)$ & Dujardin et al., 2002 \\
\hline 104 & Triatoma incrassata & Not described & \\
\hline 105 & Triatoma indictiva & Not described & \\
\hline 106 & Triatoma infestans & $2 n=22(20 A+X Y)$ & Schreiber and Pellegrino, 1950 \\
\hline 107 & Triatoma i. melanosoma & $2 n=22(20 A+X Y)$ & Panzera et al., 1996 \\
\hline 108 & Triatoma jatai & Not described & \\
\hline 109 & Triatoma juazeirensis & $2 n=22(20 A+X Y)$ & Panzera et al., 2000 \\
\hline 110 & Triatoma jurbergi & $2 n=22(20 A+X Y)$ & Dujardin et al., 2002 \\
\hline 111 & Triatoma klugi & $2 n=22(20 A+X Y)$ & Costa et al., 2008 \\
\hline 112 & Triatoma lecticularia & $2 n=22(20 A+X Y)$ & Ueshima, 1966 \\
\hline 113 & Triatoma lenti & $2 n=22(20 A+X Y)$ & Alevi et al., 2012b \\
\hline 114 & Triatoma leopoldi & Not described & \\
\hline 115 & Triatoma limai & Not described & \\
\hline 116 & Triatoma maculata & $2 n=22(20 A+X Y)$ & Schreiber and Pellegrino, 1950 \\
\hline 117 & Triatoma matogrossensis & $2 n=22(20 A+X Y)$ & Crossa et al., 1992 \\
\hline 118 & Triatoma melanica & $2 \mathrm{n}=22(20 \mathrm{~A}+\mathrm{XY})$ & Panzera et al., 2000 \\
\hline 119 & Triatoma melanocephala & $2 n=24\left(20 A+X_{1} X_{2} X_{3} Y\right)$ & Alevi et al., 2012b \\
\hline 120 & Triatoma mexicana & $2 \mathrm{n}=23\left(20 \mathrm{~A}+\mathrm{X}_{1} \mathrm{X}_{2} \mathrm{Y}\right)$ & Panzera et al., 2010 \\
\hline 121 & Triatoma migrans & Not described & \\
\hline 122 & Triatoma neotomae & Not described & \\
\hline 123 & Triatoma nigromaculata & Not described & \\
\hline 124 & Triatoma nitida & $2 n=21\left(18 A+X_{1} X_{2} Y\right)$ & Schreiber and Pellegrino, 1950 \\
\hline 125 & Triatoma oliveirai & Not described & \\
\hline 126 & Triatoma patagonica & $2 n=22(20 A+X Y)$ & Ueshima, 1966 \\
\hline 127 & Triatoma peninsularis & $2 \mathrm{n}=23\left(20 \mathrm{~A}+\mathrm{X}_{1} \mathrm{X}_{2} \mathrm{Y}\right)$ & Ueshima, 1966 \\
\hline 128 & Triatoma petrochiae & $2 n=22(20 A+X Y)$ & Panzera et al., 2000 \\
\hline 129 & Triatoma pintodiasi & $2 n=22(20 A+X Y)$ & Present study \\
\hline 130 & Triatoma platensis & $2 n=22(20 A+X Y)$ & Schreiber and Pellegrino, 1950 \\
\hline 131 & Triatoma protracta & $2 n=23\left(20 A+X_{1} X_{2} Y\right)$ & Ueshima, 1966 \\
\hline 132 & Triatoma pugasi & Not described & \\
\hline 133 & Triatoma pseudomaculata & $2 n=22(20 A+X Y)$ & Schreiber et al., 1972 \\
\hline 134 & Triatoma recurva & Not described & \\
\hline 135 & Triatoma rubida & $2 n=23\left(20 A+X_{1} X_{2} Y\right)$ & Ueshima, 1966 \\
\hline 136 & Triatoma rubrofasciata & $2 \mathrm{n}=25\left(22 \mathrm{~A}+\mathrm{X}_{1} \mathrm{X}_{2} \mathrm{Y}\right)$ & Manna, 1950 \\
\hline 137 & Triatoma rubrovaria & $2 n=22(20 A+X Y)$ & Schreiber and Pellegrino, 1950 \\
\hline 138 & Triatoma ryckmani & $2 n=23\left(20 \mathrm{~A}+\mathrm{X}_{1} \mathrm{X}_{2} \mathrm{Y}\right)$ & Dujardin et al., 2002 \\
\hline 139 & Triatoma sanguisuga & $2 n=23\left(20 A+X_{1} X_{2} Y\right)$ & Payne, 1909 \\
\hline 140 & Triatoma sherlocki & $2 n=22(20 A+X Y)$ & Panzera et al., 2010 \\
\hline 141 & Triatoma sinaloensis & $2 n=23\left(20 A+X_{1} X_{2} Y\right)$ & Ueshima, 1966 \\
\hline 142 & Triatoma sinica & Not described & \\
\hline 143 & Triatoma sordida & $2 n=22(20 A+X Y)$ & Schreiber and Pellegrino, 1950 \\
\hline 144 & Triatoma tibiamaculata & $2 n=22(20 A+X Y)$ & Panzera et al., 1998 \\
\hline 145 & Triatoma vandae & $2 n=22(20 A+X Y)$ & Panzera et al., 2010 \\
\hline 146 & Triatoma venosa & Not described & \\
\hline 147 & Triatoma vitticeps & $2 \mathrm{n}=24\left(20 \mathrm{~A}+\mathrm{X}_{1} \mathrm{X}_{2} \mathrm{X}_{3} \mathrm{Y}\right)$ & Schreiber and Pellegrino, 1950 \\
\hline 148 & Triatoma williami & $2 \mathrm{n}=22(20 \mathrm{~A}+\mathrm{XY})$ & Dujardin et al., 2002 \\
\hline 149 & Triatoma wygodzinskyi & $2 n=22(20 A+X Y)$ & Panzera et al., 2012 \\
\hline 150 & Triatoma dominicana & Not described & \\
\hline
\end{tabular}




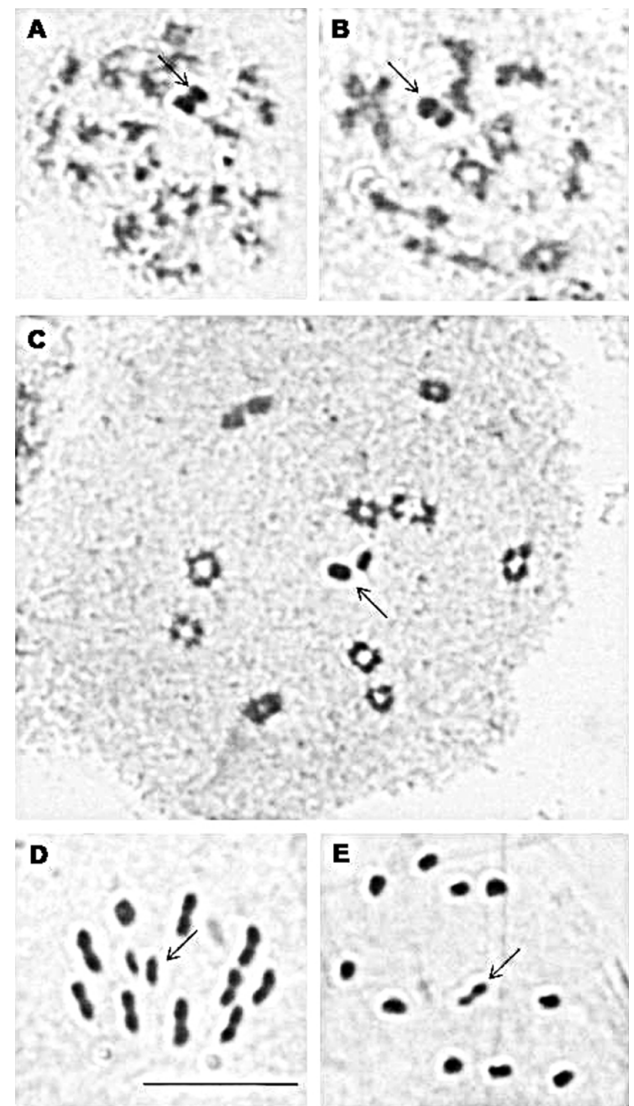

Figure 1. Meiosis of Triatoma pintodiasi. Diffuse intermediate stage (zygotene/pachytene) (A and $\mathbf{B}$ ); diffuse final stage (diplotene) (C) with chromocenter that made it possible to distinguish the two sex chromosomes (arrows). Metaphase I (D) and metaphase II (E) with 22 chromosomes (20 autosomes + XY). Note the more heteropyknotic Y sex chromosome (arrows). Bar: $10 \mu \mathrm{m}$.
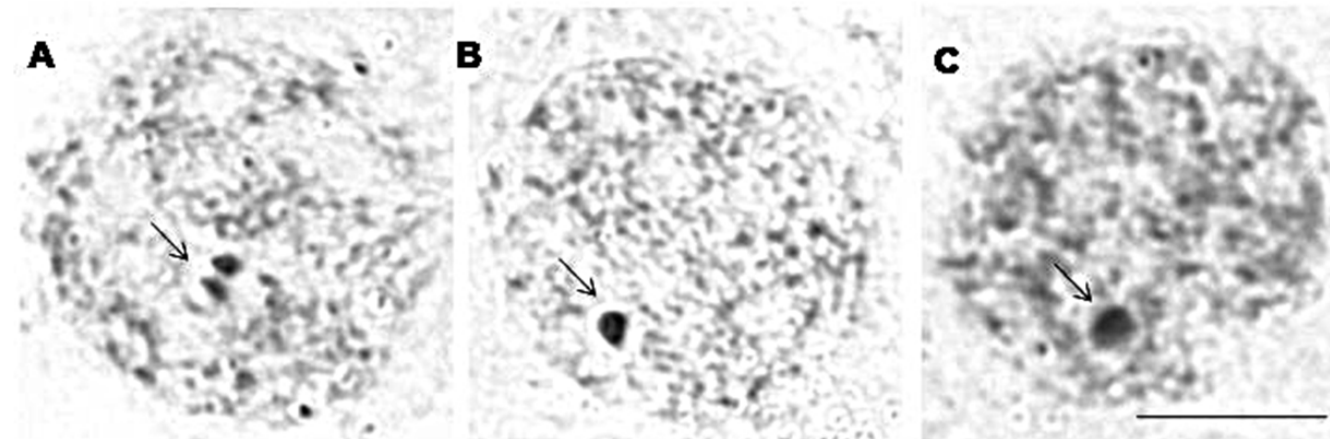

Figure 2. Prophase (initial diffuse stage) of Triatoma pintodiasi (A), T. carcavalloi (B), and T. circummaculata (C). Note that $T$. pintodiasi presented a chromocenter that made it possible to distinguish the two sex chromosomes (A; arrow), while T. carcavalloi (B; arrow), and T. circummaculata (C; arrow) showed a single chromocenter formed by the sex chromosomes. Bar: $10 \mu \mathrm{m}$. 
Ueshima (1979) suggested that the common ancestor of the triatomines showed 22 chromosomes. In addition, Nokkala and Nokkala $(1983,1984)$ believe that the common ancestor of the Hemiptera order had system sex determination of type XY. Thus, based on the principle that the common ancestor has 22 chromosomes, we suggest that major events that occurred during the karyotype evolution of these insects included mainly agmatoploidy (fission) and simploidy (fusion).

Cytogenetic analyses of triatomines are of great importance, because chromosome data, characteristics of spermatids, and meiotic features can be applied to determine cytotaxonomy of these vectors. Analysis of early prophase for example, can aid in differentiation between $T$. sordida and T. guasayana (Rebagliati et al., 1998). Cytogenetic data have also assisted in the revalidation of $T$. garciabesi (Jurberg et al., 1998); in the description of Mepraia parapatrica (Frías-Lasserre, 2010); and more recently, by analysis of spermatids, in the differentiation of morphologically related species (Alevi et al., 2013b, 2014b).

The present study describes initial cytogenetic characteristics of $T$. pintodiasi and suggests differences in the chromocenter, as a possible cytotaxonomic tool. The authors emphasize the need for further cytogenetic, molecular, biological, and biogeographical analysis, in addition to experimental hybrid crosses with other species of the Rubrovaria subcomplex.

\section{Conflicts of interest}

The authors declare no conflict of interest.

\section{ACKNOWLEDGMENTS}

Research supported by Fundação de Amparo à Pesquisa do Estado de São Paulo (FAPESP; Process \#2013/19764-0) and Conselho Nacional de Desenvolvimento Científico e Tecnológico (CNPq).

\section{REFERENCES}

Abad-Franch F, Pavan MG, Jaramillo-O N, Palomeque FS, et al. (2013). Rhodnius barretti, a new species of Triatominae (Hemiptera: Reduviidae) from western Amazonia. Mem. Inst. Oswaldo Cruz 108 (Suppl 1): 92-99.http://dx.doi. org/10.1590/0074-0276130434

Alevi KCC, Mendonça PP, Pereira NP, Rosa JA, et al. (2012a). Karyotype of Triatoma melanocephala Neiva and Pinto (1923). Does this species fit in the Brasiliensis subcomplex? Infect. Genet. Evol. 12: 1652-1653.http://dx.doi. org/10.1016/j.meegid.2012.06.011

Alevi KCC, Mendonça PP, Succi M, Pereira NP, et al. (2012b). Karyotype and spermatogenesis in Triatoma lenti (Hemiptera: Triatominae), a potential Chagas vector. Genet. Mol. Res. 11: 4278-4284.http://dx.doi.org/10.4238/2012. December. 17.3

Alevi KCC, Rosa JA and Azeredo Oliveira MTV (2013a). Mini Review: karyotypic survey in Triatominae subfamily (Hemiptera, Heteroptera). Entomol. Ornithol. Herpetol. 2: 106. http://dx.doi.org/10.4172/2161-0983.1000106

Alevi KCC, Mendonça PP, Pereira NP, Fernandes ALVZ, et al. (2013b). Analysis of spermiogenesis like a tool in the study of the triatomines of the Brasiliensis subcomplex. C. R. Biol. 336: 46-50.http://dx.doi.org/10.1016/j.crvi.2013.01.005

Alevi KCC, da Costa Castro NF, Lima AC, Ravazi A, et al. (2014a). Nucleolar persistence during spermatogenesis of the genus Rhodnius (Hemiptera, Triatominae). Cell Biol. Int. 38: 977-980.http://dx.doi.org/10.1002/cbin.10297

Alevi KCC, Mendonça PP, Pereira NP and Rosa JA (2014b). Heteropyknotic filament in spermatids of Triatoma melanocephala and T. vitticeps (Hemiptera, Triatominae). Inv. Rep. Dev. 58: 9-12. http://dx.doi.org/10.1080/0792 $\underline{4259.2013 .793623}$

Alevi KCC, Ravazi A, Mendonça VJ, Rosa JA, et al. (2015a). Karyotype of Rhodnius montenegrensis (Hemiptera, Triatominae). Genet. Mol. Res. 14: 222-226.http://dx.doi.org/10.4238/2015.January.16.5 
Alevi KCC, Reis YV, Borgueti AO, Mendonça VJ, et al. (2015b). Diploid chromosome set of kissing bug Triatoma baratai (Hemiptera, Triatominae). Genet. Mol. Res. 14: 1106-1110.http://dx.doi.org/10.4238/2015.February.6.14

Almeida CE, Marcet PL, Gumiel M, Takiya DM, et al. (2009). Phylogenetic and phenotypic relationships among Triatoma carcavalloi (Hemiptera: Reduviidae: Triatominae) and related species collected in domiciles in Rio Grande do Sul State, Brazil. J. Vector Ecol. 34: 164-173.http://dx.doi.org/10.1111/j.1948-7134.2009.00023.x

Barth R (1956). Estudos anatômicos e histológicos sôbre a subfamília Triatominae (Hemiptera, Reduviidae). VI. Estudo comparativo sôbre a espermiocitogênese das espécies mais importantes. Mem. Inst. Oswaldo Cruz 54: 599-623.http:// dx.doi.org/10.1590/S0074-02761956000300009

Costa LC, Azeredo-Oliveira MTV and Tartarotti E (2008). Spermatogenesis and nucleolar activity in Triatoma klugi (Triatominae, Heteroptera). Genet. Mol. Biol. 31: 438-444. http://dx.doi.org/10.1590/S1415-47572008000300008

Crossa RP, Hernández M, Caraccio MN, Rose V, et al. (2002). Chromosomal evolution trends of the genus Panstrongylus (Hemiptera, Reduviidae), vectors of Chagas disease. Infect. Genet. Evol. 2: 47-56.http://dx.doi.org/10.1016/S15671348(02)00063-1

de Lucena DT (1970). Estudos sôbre a doença de Chagas no nordeste do Brasil. Rev. Bras. Malariol. Doenças Trop. 22: 3-173.

De Vaio ES, Grucci B, Castagnino AM, Franca ME, et al. (1985). Meiotic differences between three triatomine species (Hemiptera, Reduviidae). Genetica 67: 185-191. http://dx.doi.org/10.1007/BF02424489

Dujardin JP, Schofield CJ and Panzera F (2002). Los vectores de la enfermedad de Chagas. Académie Royale des Science d'Outre Mer, Brussels, Belgium.

Frías D, Henry A and González CH (1998). Mepraia gajardoi: a new species of Triatominae (Hemíptera: Reduviidae) from Chile and its comparison with Mepraia spinolai. Rev. Chil. Hist. Nat. 71: 177-188.

Frías-Lasserre D (2010). A new species and karyotype variation in the bordering distribution of Mepraia spinolai (Porter) and Mepraia gajardoi Frías et al (Hemiptera: Reduviidae: Triatominae) in Chile and its parapatric model of speciation. Neotrop. Entomol. 39: 572-583. http://dx.doi.org/10.1590/S1519-566X2010000400017

Gardim S, Rocha CS, Almeida CE, Takiya DM, et al. (2013). Evolutionary relationships of the Triatoma matogrossensis subcomplex, the endemic Triatoma in Central-Western Brazil, based on mitochondrial DNA sequences. Am. J. Trop. Med. Hyg. 89: 766-774. http://dx.doi.org/10.4269/ajtmh.12-0718

Gómez-Palacio A, Jaramillo-Ocampo N, Triana-Chávez O, Saldaña A, et al. (2008). Chromosome variability in the Chagas disease vector Rhodnius pallescens (Hemiptera, Reduviidae, Rhodniini). Mem. Inst. Oswaldo Cruz 103: 160-164. http://dx.doi.org/10.1590/S0074-02762008000200006

Hwang WS and Weirauch C (2012). Evolutionary history of assassin bugs (Insecta: Hemiptera: Reduviidae): insights from divergence dating and ancestral state reconstruction. PLoS One 7: e45523. http://dx.doi.org/10.1371/journal. pone. 0045523

Jurberg J, Galvão C, Lent H, Monteiro F, et al. (1998). Revalidação de Triatoma garciabesi Carcavallo, Cichero, Martínez, Prosen \& Ronderos (1967) (Hemiptera-Reduviidae). Entomol. Vect 5: 107-122.

Jurberg J, Cunha V, Cailleaux S, Raigorodschi R, et al. (2013). Triatoma pintodiasi sp. nov. do subcomplexo T. rubrobaria (Hemiptera, Reduviidae, Triatominae). Rev. Pan.-Amaz. Saúde 4: 43-56.

Justi SA, Russo CAM, Mallet JRS, Obara MT, et al. (2014). Molecular phylogeny of Triatomini (Hemiptera: Reduviidae: Triatominae). Parasit. Vectors 7: 149. http://dx.doi.org/10.1186/1756-3305-7-149

Koshy TK (1979a). Chromosomes of Triatominae I: Haploid karyotypes of three species in the genus Rhodnius (hemiptera: Reduviidae). Acta Cient. Venez. 30: 183-190.

Koshy TK (1979b). Chromosomes of Triatominae II: Karyotypes studies of five species in the genus Rhodnius (Hemiptera: reduviidae). Acta Cient. Venez. 30: 191-195.

Manna GK (1950). Multiple sex chromosome mechanism in a reduviid bug Conorhinus rubrofasciata (De Geer). Proc. Zool. Soc. Bengal. 3: 155-161.

Nokkala S and Nokkala C (1983). Achiasmatic male meiosis in two species of Saldula (Saldidae, Hemiptera). Hereditas 99: 131-134. http://dx.doi.org/10.1111/j.1601-5223.1983.tb00737.x

Nokkala S and Nokkala C (1984). Achiasmatic male meiosis in the Heteropteran genus Nabis (Nabidae, Hemiptera). Hereditas 101: 31-35. http://dx.doi.org/10.1111/j.1601-5223.1984.tb00445.x

Panzera F, Pérez R, Hornos S, Panzera Y, et al. (1996). Chromosome numbers in the Triatominae (Hemiptera-Reduviidae): a review. Mem. Inst. Oswaldo Cruz 91: 515-518. http://dx.doi.org/10.1590/S0074-02761996000400021

Panzera F, Hornos S, Pereira J, Cestau R, et al. (1997). Genetic variability and geographic differentiation among three species of Triatomine bugs (Hemiptera-Reduviidae). Am. J. Trop. Med. Hyg. 57: 732-739.

Panzera F, Scvortzoff E, Pérez R, Panzera Y, et al. (1998). Cytogenetics of Triatomines. In: Atlas of Chagas disease vectors in the Americas (Carcavallo RU, Galíndez-Girón I, Jurberg J and Lent H, eds.). Editora Fiocruz, Rio de Janeiro, 621-664.

Panzera F, Pérez R, Nicolini P, Hornos S, et al. (2000). Chromosome homogeneity in populations of Triatoma brasiliensis Neiva 1911 (Hemiptera - Reduviidae - Triatominae). Cad. Saude Publica 16 (Suppl 2): 83-88.http://dx.doi. org/10.1590/S0102-311X2000000800009 
Panzera F, Pérez R, Panzera Y, Ferrandis I, et al. (2010). Cytogenetics and genome evolution in the subfamily Triatominae (Hemiptera, Reduviidae). Cytogenet. Genome Res. 128: 77-87. http://dx.doi.org/10.1159/000298824

Panzera Y, Pita S, Ferreiro MJ, Ferrandis I, et al. (2012). High dynamics of rDNA cluster location in kissing bug holocentric chromosomes (Triatominae, Heteroptera). Cytogenet. Genome Res. 138: 56-67. http://dx.doi.org/10.1159/000341888

Payne F (1909). Some new types of chromosome distribution and their relation to sex. Biol. Bull. 16: 119-166. http:// dx.doi.org/10.2307/1536127

Pérez R, Panzera Y, Scafiezzo S, Mazzella MC, et al. (1992). Cytogenetics as a tool for triatomine species distinction (Hemiptera-Reduviidae). Mem. Inst. Oswaldo Cruz 87: 353-361. http://dx.doi.org/10.1590/S0074$\underline{02761992000300004}$

Poinar G Jr (2013). Panstrongylus hispaniolae sp. n. (Hemiptera: Reduviidae: Triatominae), a new fossil triatomine in Dominican amber, with evidence of gut flagellates. Palaeodiversity 6: 1-8.

Rebagliati P, Papeschi AG, Mola LM, Pietrokovsky S, et al. (1998). Comparative meiotic studies in Triatoma sordida (Stål) and T. guasayana Wygodzinsky \& Abalos (Reduviidae, Heteroptera). Mem. Inst. Oswaldo Cruz 93: 309-315. http://dx.doi.org/10.1590/S0074-02761998000300007

Santos SM (2010). Estudo citogenético de quatro espécies de triatomínae (Hemiptera: Reduviidae). Doctoral thesis, Universidade Federal de Viçosa, Minas Gerais, Brasil.

Schofield CJ and Galvão C (2009). Classification, evolution, and species groups within the Triatominae. Acta Trop. 110: 88-100. http://dx.doi.org/10.1016/j.actatropica.2009.01.010

Schreiber G and Pellegrino J (1950). Eteropicnosi di autosomi come possibile meccanismo di speciazione; ricerche citologiche su alcuni Emitteri neotropici. Sci Genet 3: 215-226.

Schreiber G, Bogliolo AR and Coelho de Pinho A (1972). Cytogenetics of Triatominae: Caryotype, DNA content, nuclear size and heteropycnosis of autosomes. Rev. Bras. Biol. 32: 255-263.

Tartarotti E and Azeredo-Oliveira MTV (1999). Patterns of nucleolar activity during spermatogenesis of two triatomines, Panstrongylus megistus and P. herreri. Caryologia 52: 177-184. http://dx.doi.org/10.1080/00087114.1998.10589171

Tartarotti E, Azeredo-Oliveira MTV and Ceron CR (2006). Phylogenetic approach to the study of triatomines (Triatominae, Heteroptera). Braz. J. Biol. 66 (2B): 703-708. http://dx.doi.org/10.1590/S1519-69842006000400014

Ueshima N (1966). Cytotaxonomy of the triatominae (Reduviidae: Hemiptera). Chromosoma 18: 97-122. http://dx.doi. org/10.1007/BF00326447

Ueshima N (1979). Insecta 6. Hemiptera II: Heteroptera. In: Animal Cytogenetics (John B, ed.). Gebruder Borntraeger, Berlin, Stuttgart, 113. 